\title{
Dependencia funcional y apoyo para personas mayores de México, 2001-2026
}

\author{
César González-González¹, Gianluca Cafagna², María del Carmen Hernández Ruiz³, Pablo Ibarrarán \\ y Marco Stampini ${ }^{4}$
}

Forma de citar

González-González C, Cafagna G, Hernández Ruiz MC, Ibarrarán P, Stampini M. Dependencia funcional y apoyo para personas mayores de México, 2001-2026. Rev Panam Salud Publica. 2021;45:e71. https://doi.org/10.26633/RPSP.2021.71

RESUMEN

Objetivos. Identificar los factores asociados con la dependencia funcional futura de las personas mayores en México, así como con recibir o no apoyo para la realización de actividades básicas de la vida diaria (ABVD), y proyectar la prevalencia de la dependencia funcional en 2026.

Métodos. Se utilizaron los datos del Estudio Nacional de Salud y Envejecimiento (ENASEM) del 2001 y las rondas de seguimiento de 2012 y 2015. Se estimó un modelo de regresión logística multinomial para analizar los factores asociados con la dependencia futura y un modelo de regresión logística para los factores asociados con recibir o no apoyo. Para las proyecciones de personas mayores en situación de dependencia en 2026 se utilizaron los datos del ENASEM del 2015 y los coeficientes estimados del modelo de dependencia futura.

Resultados. Las personas de más edad, las que tenían un nivel de educación más bajo, las que padecían de hipertensión, artritis, diabetes, las que habían sufrido una embolia cerebral o alguna caída, y las que tenían algún nivel de dependencia funcional previa presentaron un riesgo significativamente mayor de dependencia (leve o severa) y de fallecer en los 11 años siguientes respecto a sus referencias. Las personas de mayor edad o con dependencia severa tuvieron mayores probabilidades de recibir apoyo respecto a sus referencias. Para el año 2026, se estima que el 18,9\% de las personas mayores en México tendrá dependencia leve y el 9,3\% dependencia severa.

Conclusiones. Los factores asociados con la dependencia futura y con fallecer fueron la edad, el nivel educacional, algunas enfermedades crónicas, haberse caído y tener dependencia funcional previa; los factores asociados con recibir apoyo para la realización de ABVD fueron tener dependencia severa y la edad. Se estima que la prevalencia de la dependencia aumentará 2,1 veces en 25 años (2001-2026).

Palabras clave Actividades cotidianas; cuidados a largo plazo; envejecimiento; México.

Debido al aumento en la esperanza de vida y la reducción en las tasas de fecundidad, los países de América Latina y el Caribe transitan por un proceso de envejecimiento de la población que trae aparejado un incremento en el número de personas en situación de dependencia funcional, es decir, que necesitan apoyo de otras personas por un período prolongado para desempeñar ciertas actividades de la vida diaria $(1,2)$.

Facultad de Trabajo Social, Universidad de Colima, Colima, México.

2 Práctica Global de Salud, Nutrición y Población, Banco Mundial, Washington, DC, Estados Unidos de América.

3 Escuela de Gobierno John F. Kennedy, Universidad de Harvard, Cambridge, Estados Unidos de América.
Diversos estudios señalan que las características demográficas y socioeconómicas, el estado de salud y cognitivo, y algunos factores de riesgo - como la obesidad o el sobrepeso, y el consumo de tabaco y alcohol- están asociados con la dependencia en personas mayores $(3,4)$.

En México, el proceso de envejecimiento demográfico presenta un ritmo acelerado (5). Se estima que en solo 22 años (del 
2018 al 2040), las personas de 65 años o más pasarán de representar el $7 \%$ de la población al $14 \%$, algo que requeriría, por ejemplo, 69 años en Estados Unidos de América y 115 años en Francia (6). Además, en México se observa una alta prevalencia de enfermedades crónicas (7), por lo que se puede esperar que el número de personas con dependencia funcional aumentará consecuentemente. Las estimaciones de los niveles de dependencia en la población de 60 años o más a partir del año 2015 varían entre $15,0 \%$ (8) y 20,1\% (9), en dependencia de la fuente de información y del indicador utilizado.

Históricamente, las personas mayores con dependencia funcional se han apoyado en las redes familiares y vecinales que, de manera solidaria, ofrecen ayuda (10). Sin embargo, México está atravesando por intensos cambios sociales y en la estructura familiar — como el menor número de hijos, el considerable aumento de la migración nacional e internacional de los miembros de la familia, las nuevas formas de cohabitación, el mayor número de divorcios y nuevas nupcias, y la mayor participación laboral de las mujeres (11) — que indican que la oferta de apoyo familiar y vecinal a las personas con dependencia se reducirá en el futuro, mientras aumenta su demanda.

Estudios recientes utilizan datos del Estudio Nacional de Salud y Envejecimiento (ENASEM) (12) sobre la dependencia para realizar las actividades básicas de la vida diaria (ABVD), a fin de analizar los niveles que alcanza ese problema en la población de personas mayores en México y los factores asociados (13-15). La medición, la proyección y la elaboración de modelos de simulación que permitan visualizar la prevalencia futura de la dependencia funcional de las personas mayores en el país son elementos esenciales para poder tomar decisiones informadas y fundamentar políticas públicas.

El objetivo de este trabajo es identificar los factores asociados con la dependencia funcional futura de las personas mayores en México, así como con recibir o no apoyo para la realización de ABVD. Además, se proyecta la prevalencia de la dependencia funcional para 2026 en México.

\section{MATERIALES Y MÉTODOS}

Se realizó una investigación longitudinal a partir de los datos del ENASEM del $2001(12)^{5}$, que incluyó una muestra representativa nacional para describir el proceso de envejecimiento, las enfermedades y la carga de la dependencia funcional de las personas mayores de 50 años en México (8).

Para este análisis se utilizó la información de la encuesta basal del año 2001 (con representación nacional de las personas nacidas en 1951 o antes) y las rondas de seguimiento realizadas en 2012 y 2015 (12). En el ENASEM del 2001 se entrevistaron 13463 personas de 50 años o más, de los cuales 8436 se entrevistaron nuevamente en 2012. De los que no se entrevistaron nuevamente en 2012, 3320 habían fallecido y 1707 no se pudieron localizar o rehusaron participar. Para mantener la representatividad de la encuesta para las personas mayores de 50 años, en 2012 la muestra se amplió con 5896 personas de 50 a 60 años.

La muestra utilizada para identificar los factores asociados con la dependencia futura (dependencia en 2012, a partir de

\footnotetext{
Se pueden consultar los detalles metodológicos y acceder a las bases de datos del estudio para 2001, 2003, 2015 y 2018 en http://www.enasem.org/Index_ Esp.aspx
}

los factores observados en 2001) estuvo compuesta por 11081 personas, incluidas las encuestadas en 2001 que fueron entrevistadas nuevamente en 2012 y las fallecidas entre esos años. Además de excluir los 1707 entrevistados en 2001 que se perdieron en el seguimiento del 2012, se excluyeron 675 registros por valores faltantes en las variables analizadas.

Para el análisis de los factores asociados con recibir o no apoyo, solo se consideraron los 2070 entrevistados en 2001 que en la entrevista del 2012 declararon tener alguna dependencia funcional; se excluyeron 7 entrevistados por valores faltantes en las variables, por lo que la muestra final estuvo compuesta por 2063 personas.

Para la proyección de la dependencia funcional se utilizaron también datos del ENASEM del 2015, que incluyó a los entrevistados que habían sobrevivido y realizado por lo menos una entrevista desde el 2001. En el ENASEM del 2015 no se incluyeron nuevos entrevistados, a diferencia del ENASEM del 2012, y en consecuencia, la muestra del 2015 incluyó 12541 entrevistados de 53 años o más. Para mantener la representatividad de las personas de 50 años o más en el 2015 en las proyecciones al 2026, se construyó un factor de corrección de la siguiente manera: primero, se calcularon los promedios de los factores para a) la población de 50 años o más en 2012 y b) la población de 53 años o más en 2012; seguidamente, se dividió el primer promedio entre el segundo para obtener el factor de corrección para la población de 53 años o más en 2015; por último, se multiplicó el factor de corrección por el promedio de cada uno de los factores de la población de 53 años o más en 2015.

Para analizar los factores asociados con la dependencia futura (dependencia en 2012 a partir de los factores observados en 2001) se empleó un modelo de regresión logística multinomial. Para el nivel de dependencia funcional en 2012 se utilizó una versión adaptada del índice de Katz (16) que considera seis ABVD: caminar de un lado al otro del cuarto, bañarse en una tina o regadera, comer (por ejemplo, cortar su propia comida), acostarse y levantarse de la cama, usar el excusado (incluido subirse, bajarse o ponerse en cuclillas), y vestirse. Cada una de estas variables se dicotomizó (0: informó no tener dificultad para realizar la actividad; 1: informó tener dificultad). El nivel de dependencia se expresó según una escala de 4 puntos, donde, 1: sin dependencia; 2: dependencia leve (dificultad con 1 o 2 ABVD); 3: dependencia severa (dificultad con 3 ABVD o más); 4: fallecido (si el encuestado murió entre 2001 y 2012). La construcción de las variables sobre el nivel de dependencia funcional varía en la literatura según las categorías y la cantidad de ABVD que se toman en consideración (17-20). En este trabajo consistentemente con lo informado en otros estudios (15) - se incluyó la muerte entre las categorías de la variable sobre el nivel de dependencia, con el fin de evitar sesgos de selección por supervivencia.

Las variables en el modelo de dependencia futura incluyeron características demográficas y socioeconómicas, enfermedades crónicas no transmisibles, algunos comportamientos y factores de riesgo, y el nivel inicial de dependencia funcional, todas según lo informado en el 2001. El nivel inicial de dependencia funcional se basó en las categorías "sin dependencia", "dependencia leve" y "dependencia severa", dado que la muestra inicial incluyó solo a las personas mayores vivas.

Las características demográficas consideradas fueron la edad (en grupos de 50-59, 60-69, 70-79, y 80 años o más) y el sexo (hombre o mujer). Las características socioeconómicas fueron 
el nivel educacional (ninguno, primaria incompleta, primaria completa, más que educación primaria), el nivel de ingreso mensual individual (menos de 1 salario mínimo, de 1 a 2,9 salarios mínimos, y 3 salarios mínimos o más), el número de hijos vivos, la zona de residencia (urbana ${ }^{6}$ o rural), el tipo de convivencia familiar (solo, con pareja, con hijos, y otro tipo de arreglo familiar). La variable convivencia familiar se construyó para identificar a las personas mayores que vivían solas o con su cónyuge o hijos, por lo que no se tuvieron en cuenta otros arreglos residenciales, como las combinaciones de yerno/nuera, nieto/ nieta, padre/madre, hermano/hermana.

Las enfermedades crónicas no transmisibles registradas fueron: artritis, ataque cardíaco, cáncer, diabetes, embolia cerebral, hipertensión y alguna enfermedad pulmonar. Los factores y comportamientos de riesgo considerados fueron el consumo de tabaco (nunca, alguna $\mathrm{vez}^{7}$, fuma actualmente), el consumo de alcohol (nunca, alguna vez o actualmente toma bebidas alcohólicas como cerveza, vino, licor o pulque), el índice de masa corporal (bajo peso o normal: $<25$, sobrepeso: $\geq 25$ y $<30$, obesidad: $\geq 30$ ) y el haberse caído durante los dos años previos a la encuesta (sí o no).

Los resultados del modelo de dependencia futura se presentan mediante cocientes de riesgo relativo (CRR), con intervalos de confianza de 95\% (IC95\%). Se estimó, además, el efecto marginal de las variables.

Para estudiar los factores asociados con recibir o no apoyo en 2012, condicionado a estar en situación de dependencia ese año, se utilizó un modelo de regresión logística. La variable recibir o no apoyo fue dicotómica (1: recibe apoyo; 2: no recibe apoyo). Se consideró que una persona recibía apoyo si alguien lo ayudaba a realizar al menos una ABVD; por definición de la variable, solo podían recibir apoyo los encuestados con dependencia funcional. No se diferenció entre el apoyo pagado y el apoyo sin pago debido a que el porcentaje de apoyo pagado en la población mexicana es muy bajo $(2,6 \%)(21)$. Las variables incluidas en el modelo de recibir o no apoyo fueron las características demográficas y socioeconómicas medidas en 2001 y el nivel de dependencia observado en 2012. Para el nivel de dependencia en 2012, se utilizaron en este modelo solo las categorías "dependencia leve" y "dependencia severa", ya que solo se consideraron las personas mayores en situación de dependencia. La inclusión en el modelo del nivel de dependencia en 2012 se debe a que se quiere analizar la asociación entre recibir (o no) apoyo y el nivel de dependencia. Se excluyeron las enfermedades crónicas y algunos factores de riesgo medidos en 2001, ya que se consideró que esas variables no influirían directamente en recibir apoyo o no, porque lo hacen por medio del nivel de dependencia observado en 2012, que fue incluido entre las variables del modelo.

Los resultados del modelo de recibir o no apoyo se presentan mediante la razón de posibilidades (OR, por el término en inglés odds ratio), con sus respectivos IC95\%. Se estimó, asimismo, el efecto marginal de las variables.

Para las proyecciones de personas mayores en situación de dependencia en el año 2026 se utilizaron los datos disponibles del ENASEM del 2015 y los coeficientes estimados del modelo de dependencia futura. El supuesto principal que respalda tales

\footnotetext{
Se consideraron urbanas las localidades con 100000 habitantes o más.

Se consideró que la persona había consumido tabaco alguna vez si había fumado más de 100 cigarros o cigarrillos en su vida.
}

proyecciones es que los coeficientes estimados en 2001-2012 se mantendrían constantes en el período 2015-2026, por lo que se podrían aplicar a los datos del 2015 para obtener las proyecciones al 2026.

\section{RESULTADOS}

\section{Descripción de la muestra estudiada}

Las características de las personas incluidas en el ENASEM se presentan en el cuadro 1 . En el año 2001, 45,5\% de los encuestados tenía entre 50 y 59 años y 7,7\% tenía 80 años o más; los hombres representaron el $46,7 \%$ de la muestra. El nivel de ingresos de la población fue en general bajo y el 66,0\% no había completado la educación primaria. El número de hijos vivos fue 1,4 en promedio. La mayoría de las personas mayores encuestadas vivían con otras personas (parejas, hijos u otro tipo de arreglo familiar), sin embargo, se debe hacer notar que 7,9\% de ellas vivía sola.

Con relación a las enfermedades crónicas, 37,2\% informó padecer de hipertensión y 15,3\% tenía diabetes. Estas prevalencias son coherentes con los factores de riesgo informados: $56,5 \%$ de sobrepeso y $15,5 \%$ de obesidad. Además, en el momento de la encuesta, 17,3\% fumaba, 30,7\% consumía alcohol y 37,2\% había sufrido al menos una caída en los dos años previos.

De los encuestados inicialmente, 6,3\% tenía dependencia leve y 3,4\% dependencia severa; en 2015 estos valores habían aumentado a $12,0 \%$ y $5,6 \%$, respectivamente.

\section{Factores asociados con tener dependencia en el futuro y fallecer}

Los resultados muestran que las personas que en 2001 tenían mayor edad, un nivel educacional más bajo y algún grado de dependencia funcional presentaron 11 años después riesgos significativamente mayores de dependencia (leve o severa) y de haber fallecido en ese intervalo de tiempo respecto a sus referencias (cuadro 2). De manera similar, las personas mayores con hipertensión, artritis, diabetes o embolia cerebral en 2001 presentaron mayor riesgo de tener algún grado de dependencia en 2012 y de haber fallecido que sus referencias sin esas enfermedades. Por ejemplo, las personas mayores con diabetes en 2001 presentaron un riesgo significativamente mayor de fallecer $(\mathrm{CRR}=4,02 ; \mathrm{IC} 95 \%: 3,51-4,61 ; p<0,01)$, tener dependencia leve $(\mathrm{CRR}=1,56$; IC95\%: 1,31-1,85; $p<0,01)$ y severa $(\mathrm{CRR}=2,43$; IC95\%: 1,95-3,02; $p<0,01$ ) en 2012 respecto a las personas mayores que no tenían diabetes en 2001 (cuadro 2). Entre los factores asociados con haber fallecido en 2012 destacan la edad (CRR hasta 53,89), tener dependencia severa (CRR hasta 9,79) y padecer diabetes (CRR hasta 4,02) en 2001. Al considerar niveles crecientes de dependencia y el haber fallecido, el impacto de cada uno de estos tres factores aumentó.

Los factores asociados con un riesgo significativamente mayor de fallecer en el intervalo de 11 años fueron vivir solo, vivir en zona urbana, y haber fumado o fumar en 2001, pero sin asociación demostrable con la dependencia funcional. Por el contrario, las personas mayores con obesidad en 2001 presentaron un riesgo significativamente mayor de tener dependencia leve (CRR = 1,679; IC95\%: 1,393-2,022; $p<0,001)$ o severa (CRR = 1,572; IC95\%: 1,211-2,040; $p<0,001$ ) en 2012, aunque no de fallecer, respecto a las personas sin obesidad (cuadro 2 ). 
CUADRO 1. Características de la población según datos del ENASEM. México, 2001 y 2015

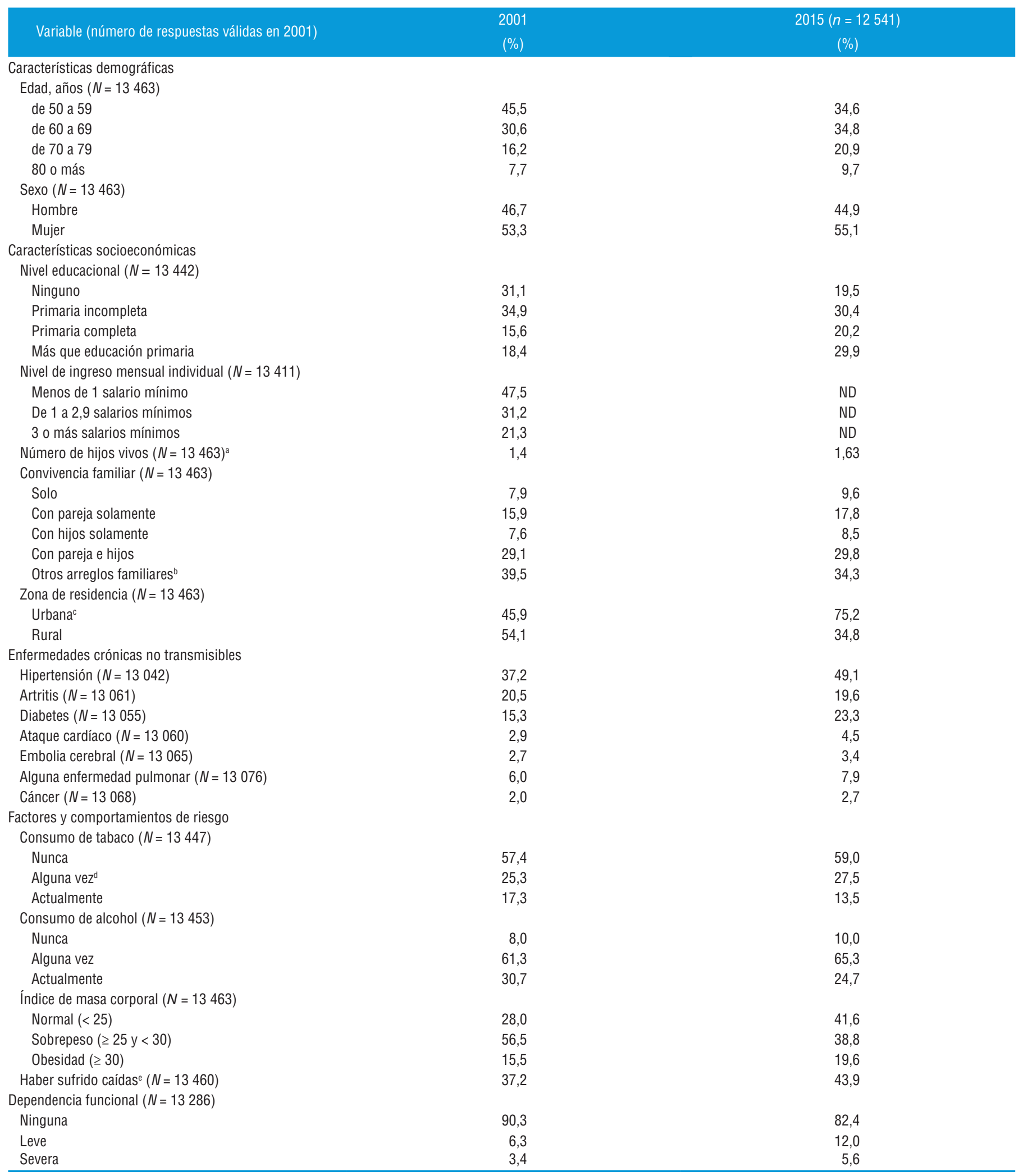

Fuente: elaborado por los autores con datos del Estudio Nacional de Salud y Envejecimiento en México (ENASEM) del año 2001 y 2015 (12).

Nota: ND: no disponible.

Se presenta la media del número de hijos vivos.

Combinaciones de yerno/nuera, nieto/nieta, padre/madre, hermano/hermana y otro conviviente no familiar, entre otros.

Se consideraron urbanas las localidades con 100000 habitantes o más.

Se consideró que la persona había consumido tabaco alguna vez si había fumado más de 100 cigarros o cigarrillos en su vida.

e Haber sufrido alguna caída en los 2 años previos a la encuesta. 
CUADRO 2. Cocientes de riesgo relativo de dependencia leve, severa y muerte en 2012 con respecto a factores presentes en 2001 $(n=11$ 081) en México

\begin{tabular}{|c|c|c|c|}
\hline \multirow{2}{*}{ Variable } & Dependencia leve & Dependencia severa & Muerte \\
\hline & CRR $(\operatorname{IC95} \%)^{\mathrm{a}}$ & CRR (IC95\%) & CRR (IC95\%) \\
\hline \multicolumn{4}{|l|}{ Características demográficas } \\
\hline \multicolumn{4}{|l|}{ Edad (años) } \\
\hline de 60 a 69 & $1,553(1,349-1,788)^{\mathrm{e}}$ & $1,771(1,429-2,195)^{\mathrm{e}}$ & $2,447(2,153-2,781)^{\mathrm{e}}$ \\
\hline de 70 a 79 & $2,472(2,028-3,012)^{\mathrm{e}}$ & $5,706(4,455-7,308)^{\mathrm{e}}$ & $10,050(8,590-11,760)^{\mathrm{e}}$ \\
\hline 80 o más & $3,881(2,442-6,168)^{\mathrm{e}}$ & $10,160(6,262-16,500)^{\mathrm{e}}$ & $53,890(38,520-75,400)^{\mathrm{e}}$ \\
\hline Mujer & Referencia & & \\
\hline \multicolumn{4}{|l|}{ Características socioeconómicas } \\
\hline \multicolumn{4}{|l|}{ Nivel educacional } \\
\hline Ninguno & Referencia & & \\
\hline Primaria incompleta & $0,826(0,709-0,962)^{c}$ & $0,758(0,615-0,934)^{d}$ & $0,872(0,764-0,997)^{\mathrm{c}}$ \\
\hline Primaria completa & $0,664(0,548-0,805)^{\mathrm{e}}$ & $0,720(0,551-0,941)^{c}$ & $0,776(0,657-0,916)^{d}$ \\
\hline 3 o más salarios mínimos & $0,799(0,685-0,931)^{d}$ & $0,879(0,710-1,088)$ & $0,801(0,703-0,913)^{d}$ \\
\hline Número de hijos vivos (promedio) & $0,944(0,896-0,996)^{c}$ & $0,975(0,901-1,055)$ & $0,937(0,893-0,984)^{d}$ \\
\hline \multicolumn{4}{|l|}{ Convivencia familiar } \\
\hline Solo & Referencia & & \\
\hline Con pareja solamente & $0,909(0,681-1,213)$ & $0,786(0,549-1,126)$ & $0,661(0,531-0,823)^{\mathrm{e}}$ \\
\hline Con hijos solamente & $0,867(0,614-1,226)$ & $0,620(0,393-0,978)^{c}$ & $0,749(0,569-0,985)^{c}$ \\
\hline Con pareja e hijos & $1,166(0,876-1,553)$ & $0,709(0,487-1,031)$ & $0,586(0,467-0,735)^{e}$ \\
\hline Otros arreglos familiares ${ }^{b}$ & $1,028(0,784-1,348)$ & $0,874(0,625-1,221)$ & $0,725(0,589-0,893)^{d}$ \\
\hline \multicolumn{4}{|l|}{ Zona de residencia } \\
\hline Urbana $^{\dagger}$ & $1,105(0,970-1,259)$ & $1,138(0,948-1,365)$ & $1,243(1,111-1,391)^{\mathrm{e}}$ \\
\hline Rural & Referencia & & \\
\hline Enfermedades crónicas no transmisibles & Referencia: sin la enfermedad & & \\
\hline Hipertensión & $1,353(1,189-1,539)^{e}$ & $1,206(1,006-1,446)^{c}$ & $1,386(1,239-1,550)^{e}$ \\
\hline Nunca & Referencia & & \\
\hline Alguna vez $z^{9}$ & $1,062(0,909-1,241)$ & $1,061(0,857-1,313)$ & $1,166(1,022-1,330)^{c}$ \\
\hline Actualmente & $1,127(0,932-1,363)$ & $1,010(0,758-1,344)$ & $1,657(1,423-1,930)^{\mathrm{e}}$ \\
\hline \multicolumn{4}{|l|}{ Consumo de alcohol } \\
\hline Nunca & Referencia & & \\
\hline Alguna vez & $0,871(0,710-1,069)$ & $1,021(0,765-1,362)$ & $1,076(0,885-1,309)$ \\
\hline Actualmente & $0,691(0,546-0,876)^{d}$ & $0,869(0,620-1,218)$ & $0,855(0,687-1,064)$ \\
\hline \multicolumn{4}{|l|}{ Índice de masa corporal } \\
\hline Normal $(<25)$ & Referencia & & \\
\hline Sobrepeso $(\geq 25 \mathrm{y}<30)$ & $1,050(0,899-1,227)$ & $1,007(0,814-1,247)$ & $0,897(0,794-1,014)$ \\
\hline Obesidad $(\geq 30)$ & $1,679(1,393-2,022)^{\mathrm{e}}$ & $1,572(1,211-2,040)^{\mathrm{e}}$ & $0,913(0,773-1,079)$ \\
\hline Haber sufrido caídas ${ }^{\mathrm{h}}$ & $1,209(1,063-1,375)^{\mathrm{e}}$ & $1,286(1,077-1,535)^{d}$ & $1,162(1,040-1,299)^{d}$ \\
\hline \multicolumn{4}{|l|}{ Dependencia funcional } \\
\hline Ninguna & Referencia & & \\
\hline Leve & $1,969(1,516-2,557)^{\mathrm{e}}$ & $3,212(2,385-4,325)^{e}$ & $2,328(1,851-2,927)^{\mathrm{e}}$ \\
\hline Severa & $2,834(1,679-4,783)^{\mathrm{e}}$ & $7,561(4,572-12,50)^{\mathrm{e}}$ & $9,795(6,457-14,86)^{\mathrm{e}}$ \\
\hline
\end{tabular}

Fuente: elaborado por los autores con datos del Estudio Nacional de Salud y Envejecimiento en México (ENASEM) de los años 2001 y 2012 (12).

CRR: cocientes de riesgo relativo; IC $95 \%$ : intervalo de confianza de $95 \%$

Combinaciones de yerno/nuera, nieto/nieta, padre/madre, hermano/hermana y otro conviviente no familiar, entre otros.

${ }_{\mathrm{d}} p \leq 0,05$.

$p \leq 0,01$.
$p \leq 0,001$.

Se consideraron urbanas las localidades con 100000 habitantes o más.

- Se consideró que la persona había consumido tabaco alguna vez si había fumado más de 100 cigarros o cigarrillos en su vida.

${ }^{\mathrm{n}}$ Haber sufrido alguna caída en los 2 años previos a la encuesta. 
CUADRO 3. Efecto marginal de la probabilidad de tener dependencia y de haber muerto en 2012, según el comportamiento de factores presentes en 2001 ( $n=11$ 081) en México

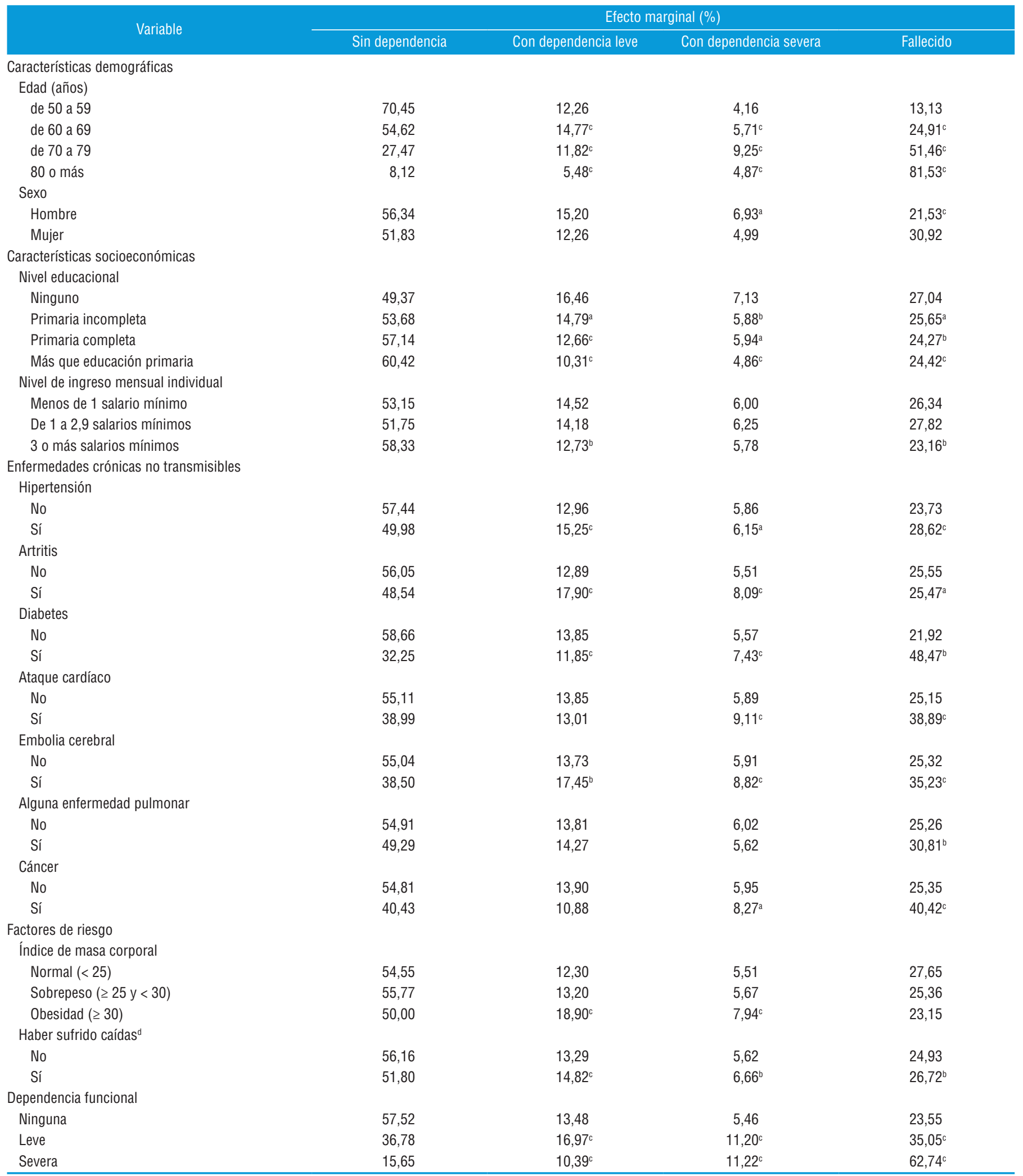

Fuente: elaborado por los autores con datos del Estudio Nacional de Salud y Envejecimiento en México (ENASEM) de los años 2001 y 2012 (12).

a $p \leq 0,05$.

${ }^{\mathrm{b}} p \leq 0,01$.
$\mathrm{c} p \leq 0,001$

a Haber sufrido alguna caída en los 2 años previos a la encuesta. 
En comparación con las mujeres, los hombres presentaron un riesgo significativamente menor de tener dependencia severa $(\mathrm{CRR}=0,783$; IC95\%: 0,630-0,972; $p<0,05)$ y mayor de fallecer $(\mathrm{CRR}=1,56$; IC95\%: 1,371-1,777; $p<0,001)$.

En el cuadro 3 se presenta el efecto marginal de las probabilidades de tener dependencia leve, severa y de haber fallecido en 2012 con respecto al comportamiento en 2001 de las variables del modelo de regresión multinomial. Por ejemplo, la probabilidad de haber fallecido entre 2001 y 2012 fue de 21,92\% en las personas mayores que no tenían diabetes en 2001, pero aumentó a $48,47 \%(p<0,01)$ en los que tenían entonces ese diagnóstico. Asimismo, las probabilidades de haber fallecido en las personas mayores con dependencia severa o leve resultaron mayores en 39,19 y 11,5 puntos porcentuales, respectivamente, $(p<0,001)$ en comparación con sus similares sin dependencia en 2001.

\section{Factores asociados con recibir apoyo}

El nivel de dependencia funcional resultó ser el principal factor asociado significativamente con recibir apoyo para la realización de ABVD en 2012. Las personas mayores con dependencia severa tuvieron más probabilidades de recibir apoyo que los que tenían dependencia leve (OR = 9,166, IC95\%: 7,3111,$49 ; p \leq 0,001$ ) (cuadro 4). El otro factor asociado con recibir apoyo fue la edad: todos los grupos de edad, a partir de los 60 años, tuvieron más probabilidades de recibir apoyo en 2012 que el grupo de 50 a 59 años. No se encontró asociación significativa entre recibir apoyo, por un lado, y la convivencia familiar, el sexo, el nivel educacional, los ingresos personales y el número de hijos vivos, por el otro.

Las personas mayores con dependencia severa presentaron una probabilidad de recibir apoyo significativamente mayor en alrededor de 50 puntos porcentuales $(p<0,001)$ respecto a los que tenían dependencia leve (76,3\% y 25,9\%, respectivamente), manteniendo las otras variables constantes en sus promedios (cuadro 4).

CUADRO 4. Razón de posibilidades y probabilidad estimada de recibir apoyo para realizar actividades básicas de la vida diaria en $2012^{\mathrm{a}}(n=2070)$ en México (2001-2012)

\begin{tabular}{|c|c|c|}
\hline Variable & $\mathrm{OR}(\mathrm{IC} 95 \%)^{\mathrm{b}}$ & $\begin{array}{l}\text { Probabilidad estimada de } \\
\text { apoyo en } 2012(\%)\end{array}$ \\
\hline \multicolumn{3}{|c|}{ Características demográficas } \\
\hline \multicolumn{3}{|c|}{ Edad en 2001 (años) } \\
\hline de 50 a 59 & Referencia & 30,0 \\
\hline de 60 a 69 & $1,551(1,213-1,983)^{c}$ & 39,9 \\
\hline de 70 a 79 & $3,724(2,762-5,022)^{c}$ & 61,4 \\
\hline 80 o más & $6,482(3,588-11,712)^{c}$ & 73,5 \\
\hline \multicolumn{3}{|c|}{ Dependencia funcional en 2012} \\
\hline Leve & Referencia & 25,9 \\
\hline Severa & $9,166(7,314-11,486)^{c}$ & 76,3 \\
\hline Constante & 0,160 & \\
\hline
\end{tabular}

Fuente: elaborado por los autores con datos del Estudio Nacional de Salud y Envejecimiento en México (ENASEM) de los años 2001 y 2012 (12).

a Controlado por sexo, nivel educacional, nivel de ingresos, número de hijos vivos y convivencia familiar en 2001.

OR: razón de posibilidades (odds ratio); IC95\%: intervalo de confianza de $95 \%$.

${ }^{c} p \leq 0,001$.

\section{Proyección de la dependencia}

A partir de los coeficientes estimados en el modelo de dependencia futura, se estimó que el 49,3\% de la población que en 2015 tenía 50 años o más no presentaría dependencia funcional en 2026, mientras que el 13,0\% tendría dependencia leve, el $6,4 \%$ dependencia severa y el 31,3\% habría fallecido para entonces (cuadro 5). Si solo se considera la población sobreviviente en 2026, el 71,8\% de la población de más de 60 años no presentaría dependencia, mientras que el 18,9\% tendría dependencia leve y el 9,3\% dependencia severa. Con respecto a la población viva en 2015, en el 2026 las mujeres tendrían una menor probabilidad de fallecer que los hombres (26,6\% contra $37,2 \%)$; mientras que $29,8 \%$ de las mujeres y $26,4 \%$ de los hombres tendrían algún tipo de dependencia.

Como es razonable que el incremento de la dependencia se deba tanto al envejecimiento de la población (factor demográfico) como al aumento de la prevalencia de enfermedades crónicas no transmisibles y otros factores de riesgo, se elaboraron proyecciones de la dependencia por grupo de edad (cuadro 5). Esas proyecciones muestran que las prevalencias de dependencia leve y severa aumentan para cada grupo de mayor edad, lo que indica que el incremento de la dependencia total no se explica solamente por el envejecimiento de la población -o porque haya más personas que presentan dependencia en los grupos de más edad- sino también por la mayor dependencia en cada grupo etario; esto sugiere que otros factores distintos de la edad son relevantes.

\section{DISCUSIÓN}

Las nuevas realidades demográficas, familiares, sociales y de salud que prevalecen en México y América Latina indican que la dependencia funcional y los servicios de apoyo a quienes están en situación de dependencia - y a sus cuidadores- son temas que deben estudiarse con mayor atención. Los resultados de este estudio ofrecen a los tomadores de decisiones un cuadro más completo sobre la dependencia en México.

En relación con los factores de dependencia futura de las personas mayores en México, los resultados destacan que tener antecedentes de dependencia, mayor edad o alguna enfermedad crónica, como la diabetes, son algunos de los factores asociados con mayores riesgos de tener dependencia leve, severa o de fallecer, respecto a sus referencias. Una primera contribución de este estudio se deriva del análisis de la relación diferenciada entre los factores analizados y cada categoría de la variable de dependencia funcional. Es decir, en este estudio se identifican los factores que incrementaron significativamente el riesgo de morir en un intervalo de 11 años, sin incrementar el riesgo de tener dependencia funcional y viceversa. Estos resultados, logrados mediante un modelo de regresión logística multinomial en el que se analizaron los factores asociados con la dependencia futura, enriquecen estudios previos basados en modelos de regresión logística ordinal $(14,15)$. Por ejemplo, se estimó que las personas mayores con obesidad en 2001 presentaron un riesgo significativamente mayor de tener dependencia pero no de haber fallecido- 11 años después respecto a las personas mayores sin obesidad en 2001, mientras que las personas mayores que fumaban en 2001 presentaron un riesgo significativamente mayor de haber fallecido - pero no de tener dependencia- en 2012, respecto a los que no fumaban en 2001. 
CUADRO 5. Características observadas (2001, 2012 y 2015) y proyectadas (2026) de la población mayor de 60 años en México, según nivel de dependencia

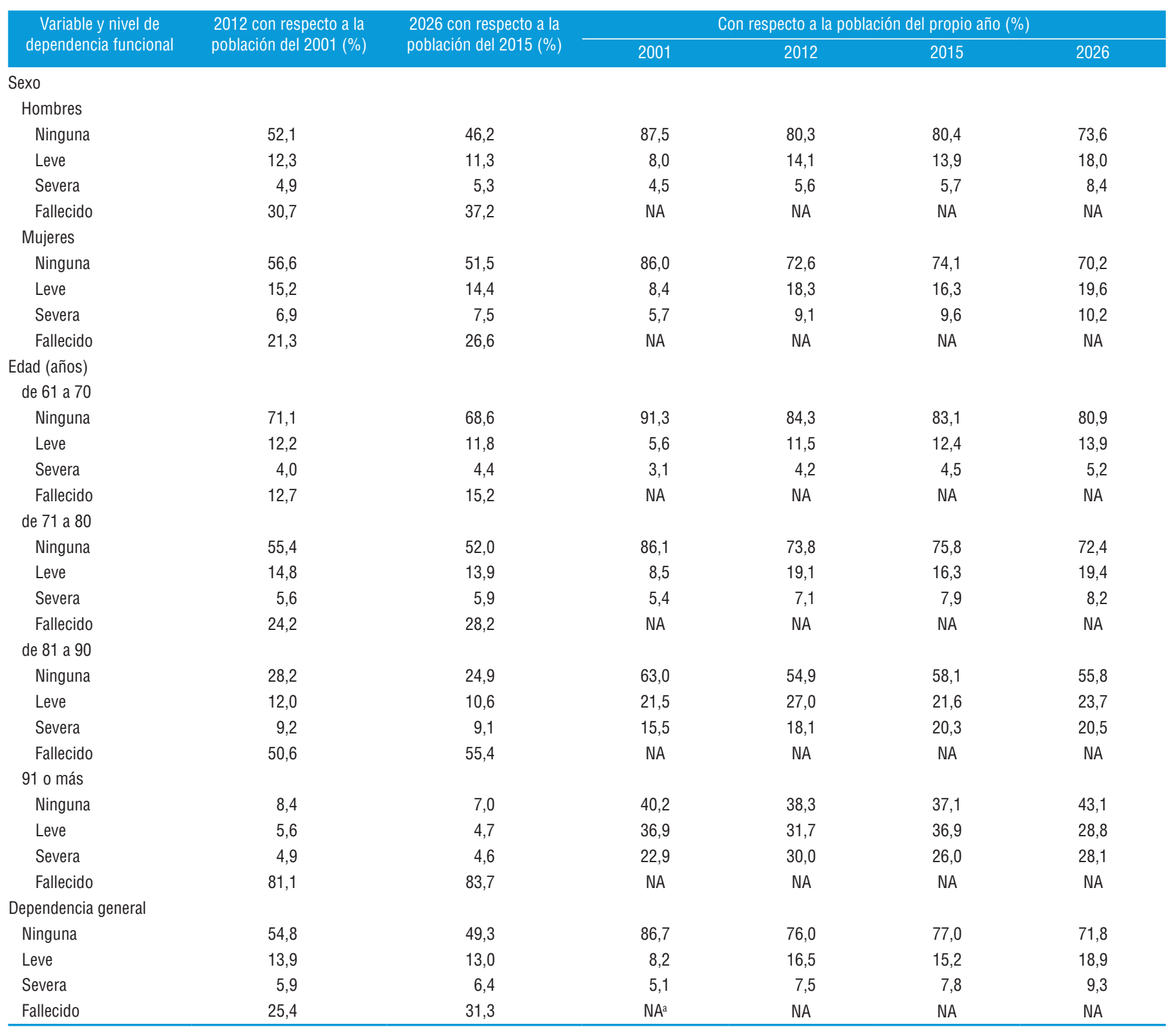

Fuente: elaboración propia con datos del Estudio Nacional de Salud y Envejecimiento en México (ENASEM) de los años 2001, 2012 y 2015 (12). Nota: NA: no aplica.

La identificación de los factores asociados con recibir o no apoyo para realizar ABVD en personas mayores en México constituye otro aporte novedoso de este estudio; aunque hay datos obtenidos en otros países (22-24) y América Latina (25, 26), este es, según nuestro mejor conocimiento, el primer estudio sobre este tema en México. Conforme a lo esperado, el nivel de dependencia funcional y la edad estuvieron significativamente asociados con recibir apoyo para la realización de ABVD; contrariamente a lo esperado, la estructura familiar (i.e., el número de hijos vivos y el tipo de convivencia familiar) no estuvo significativamente asociada con recibir apoyo. Este último resultado se puede explicar por las particularidades culturales: México es un país centrado en la familia e, independientemente de la estructura familiar, los miembros de las familias suelen ofrecer servicios de apoyo informales a sus personas mayores. Esta posible explicación es respaldada por los resultados de un análisis comparativo de los servicios de apoyo en España, Inglaterra y los Estados Unidos (22): España, el país culturalmente más centrado en la familia, fue el único en el que las variables relacionadas con la estructura familiar no estuvieron asociadas con recibir apoyo. Por otro lado, Lima-Costa y colaboradores (26) realizaron un estudio en Brasil que encontró una asociación significativa entre el número de personas en el núcleo familiar y recibir apoyo. Esta heterogeneidad en los resultados puede 
explicarse por las diferencias en los diseños (por ejemplo, el estudio de Lima-Costa y colaboradores es transversal) y por las distintas variables consideradas (el estudio citado no incluyó variables sobre el nivel de dependencia y el nivel educacional).

Por último, en relación con las proyecciones al 2026, los resultados de esta investigación confirman que el número de personas de 60 años o más con dependencia funcional continuará aumentando en los próximos años y llegará a duplicarse - y más- en 2026 respecto a lo observado en 2001. Este incremento será resultado no solo del envejecimiento poblacional, sino también del aumento de las enfermedades crónicas no transmisibles (como diabetes, artritis y embolia cerebral) y la obesidad, entre otros factores. Las proyecciones del incremento de la dependencia funcional en México están en consonancia con lo previsto para varios países de América Latina y el Caribe y apuntan a la necesidad de ampliar los servicios de apoyo a las personas mayores con ese tipo de dependencia, que se hará cada vez más apremiante en los próximos años $(2,6)$. En vista de lo anterior, es razonable pensar que los servicios informales ofrecidos por las familias podrán no ser suficientes para atender la demanda de ayuda en el futuro inmediato y será necesario complementarlos con servicios formales, cada vez más estructurados en un sistema de atención a la dependencia (6).

$\mathrm{Al}$ analizar estos resultados se deben tener en cuenta las limitaciones que impone haber empleado una definición operacional no estándar de la dependencia; además, las proyecciones suponen que los coeficientes estimados en los modelos de dependencia futura para el período 2001-2012 se mantendrán constantes en el período 2015-2026. Ambas limitaciones podrían introducir sesgos en los resultados y las conclusiones obtenidas. No obstante, se debe destacar la fortaleza del análisis por el uso de información longitudinal que permitió analizar la dependencia a lo largo de 25 años (2001-2026).

En conclusión, los factores significativamente asociados con un mayor riesgo de tener dependencia (leve o severa) en el futuro o de fallecer fueron la edad, el nivel educacional, algunas enfermedades crónicas (hipertensión, artritis, diabetes y embolia cerebral), haberse caído y tener dependencia funcional previa. Los factores significativamente asociados con recibir apoyo para la realización de ABVD fueron la edad y tener dependencia severa. Se estima que la prevalencia de dependencia total aumentará 2,1 veces en 25 años (2001-2026).

Resultados como estos pueden servir de insumos para el diseño de intervenciones y políticas públicas dirigidas a prevenir la dependencia funcional de las personas mayores o reducir su severidad, y mejorar la atención a las personas mayores que ya tienen algún grado de dependencia funcional. Se deben destinar más recursos al estudio de este problema y establecer indicadores estándar que reflejen mejor tanto la dependencia funcional como los servicios de apoyo disponibles, a fin de contar con información precisa y comparable entre países. Se recomienda continuar los esfuerzos en la generación de información sobre este tema mediante estudios longitudinales.

Contribución de los autores. CGG, GC, PI y MS contribuyeron a la concepción del manuscrito, el análisis y la interpretación de los datos, así como a la redacción y revisión crítica del manuscrito. MCHR contribuyó con la revisión crítica del manuscrito. Todos los autores revisaron y aprobaron la versión final del documento.

\section{Conflicto de intereses. Ninguno a declarar.}

Financiación. Se recibió apoyo en recursos del Banco Interamericano de Desarrollo (RG-E1656).

Declaración. Las opiniones expresadas en este artículo son responsabilidad de los autores y no necesariamente reflejan las opiniones, políticas o posiciones oficiales de las instituciones a las cuales están afiliados, ni los criterios ni la política de la Revista Panamericana de Salud Pública / Pan American Journal of Public Health y/o de la Organización Panamericana de la Salud.

\section{REFERENCIAS}

1. World Health Organization. World report on ageing and health. Luxemburgo: WHO; 2015.

2. Cafagna G, Ibarrarán P, Oliveri ML, Aranco N, Medellín N, Stampini M. Envejecer con cuidado. Atención a la dependencia en América Latina. Washington, DC: Banco Interamericano de Desarrollo; 2019. (Monografía 745) [acceso el 11 de noviembre del 2020]. Disponible en: http:/ /dx.doi.org/10.18235/0001972

3. Freitas RS, Fernandes MH, Coqueiro RS, Reis Junior W, Rocha SV, Brito TA. Functional capacity and associated factors in the elderly: A population study. Act Paul Enferm. 2012;25(6):933-9 [acceso el 11 de noviembre del 2020]. Disponible en: http:/ / www.scielo.br/pdf/ ape/v25n6/en_v25n6a17.pdf

4. Storeng SH, Sund ER, Krokstad S. Factors associated with basic and instrumental activities of daily living in elderly participants of a population-based survey: The Nord-Trøndelag Health Study, Norway. Br Med J Open. 2018;8(3):1-10.

5. González-González C, Palloni A, Wong R. Mortality and its association with chronic and infectious diseases in Mexico: A panel data analysis of older adults. Salud Publica Mex. 2015;57(supl 1):S39-45.

6. Aranco N, Stampini M, Ibarrarán P, Medellín N. Panorama de envejecimiento y dependencia en América Latina y el Caribe. Washington, DC: Banco Interamericano de Desarrollo; 2018. (Resumen de Políticas 273) [acceso el 11 de noviembre del 2020]. Disponible en: http://dx.doi.org/10.18235/0000984
7. Córdova-Villalobos JA, Barriguete-Meléndez JA, Lara-Esqueda A, Barquera S, Rosas-Peralta M, Hernández-Ávila M, et al. Las enfermedades crónicas no transmisibles en México: sinopsis epidemiológica y prevención integral. Salud Publica Mex. 2008;50(5):419-27.

8. Wong R, Michaels-Obregón A, Palloni A, Gutiérrez-Robledo LM, González-González C, López-Ortega M, et al. Progression of aging in Mexico: The Mexican Health and Aging Study (MHAS) 2012. Salud Publica Mex. 2015;57:S79-89.

9. Lozano Keymolen D, Montoya Arce BJ, Arce M. Dependencia funcional y vulnerabilidad social en adultos mayores mexicanos, 2012. Rev Latinoam Poblac. 2017;11(21):47-70.

10. Gascón SE, Redondo N. Calidad de los servicios de largo plazo para personas adultas mayores con dependencia. Nueva York: Naciones Unidas; 2014. (Serie Políticas Sociales 207) [acceso el 11 de noviembre del 2020]. Disponible en: https://repositorio.cepal.org/bitstream/ handle/11362/36948/S1420237_es.pdf?sequence=1\&isAllowed =y

11. Stampini M, Oliveri ML, Ibarrarán P, Londoño D, Rhee HJ, James GM. ¿Trabajar menos para cuidar a los padres? Los efectos laborales de la atención a la dependencia en el hogar en América Latina. Washington, DC: Banco Interamericano de Desarrollo; 2020. (Documento de Trabajo 1105) [acceso el 11 de noviembre del 2020]. Disponible en: https://publications.iadb.org/publications/spanish/document/ Trabajar-menos-para-cuidar-de-los-padres-Los-efectos-laborales-dela-atencion-a-la-dependencia-en-el-hogar-en-America-Latina.pdf 
12. Estudio Nacional de Salud y Envejecimiento en México, ENASEM. Archivos de datos y documentación (uso público). Bases de datos de los estudios de 2001, 2012 y 2015 [acceso el 29 de noviembre del 2019]. Disponible en: www.ENASEM.org

13. Kumar A, Karmarkar AM, Tan A, Graham JE, Arcari CM, Ottenbacher KJ, et al. The effect of obesity on incidence of disability and mortality in Mexicans aged 50 years and older. Salud Publica Mex. 2015;57:S31-8.

14. Díaz-Venegas C, De la Vega S, Wong R. Transitions in activities of daily living in Mexico, 2001-2012. Salud Publica Mex. 2015;57(1):S54-61.

15. Díaz-Venegas C, Reistetter TA, Wong R. Differences in the progression of disability: A U.S.-Mexico comparison. J Gerontol Psychol Sci Soc. 2016;73(5):913-22.

16. Katz S, Ford AB, Moskovitz RW, Jackson BA, Jaffe MV. Studies of illness in the aged. The index of ADL: A standardized measure of biological and psychosocial function. J Am Med Assoc. 1963;185:914-9.

17. Kim DH, Newman AB, Lipsitz LA. Prediction of severe, persistent activity-of-daily-living disability in older adults. Am J Epidemiol. 2013;178(7):1085-93.

18. Millán-Calenti JC, Tubío J, Pita-Fernández S, González-Abraldes I, Lorenzo T, Fernández-Arruty T, et al. Prevalence of functional disability in activities of daily living (ADL), instrumental activities of daily living (IADL) and associated factors, as predictors of morbidity and mortality. Arch Gerontol Geriatr. 2010;50(3):306-10.

19. Shelkey M, Wallace M. Katz index of independence in activities of daily living (ADL). Vol. 8. Try this: best practices in nursing care to older adults. New York: The Hartford Institute for Geriatric Nursing; 2012 [acceso el 11 de noviembre del 2020]. Disponible en https://www.alz.org/careplanning/downloads/katz-adl.pdf

20. Neumann LTV, Albert SM. Aging in Brazil. Gerontologist. 2018;58(4):611-7.

21. González-González C, Stampini M, Cafagna G, Hernández Ruiz MC, Ibarrarán P. Simulaciones del costo de un sistema de apoyo para los adultos mayores en situación de dependencia en México Washington, DC: Banco Interamericano de Desarrollo; 2019. (Documento de Trabajo del BID No. IDB-WP-1033) [acceso el 11 de noviembre del 2020]. Disponible en: https://publications.iadb. org/publications/spanish/document/Simulaciones_del_costo_de _un_sistema_de_apoyo_para_los_adultos_mayores_en_situaci\% C3\%B3n_de_dependencia_en_M\%C3\%A9xico_es.pdf

22. Solé-Auró A, Crimmins EM. Who cares? A comparison of informal and formal care provision in Spain, England and the USA. Ageing Soc. 2014;34(3):495-517.

23. Van Groenou M, Glaser K, Tomassini C, Jacobs T. Socio-economic status differences in older people's use of informal and formal help: A comparison of four European countries. Ageing Soc. 2006;26(5):745-66.

24. Pego MA, Nunes C. Aging, disability, and informal caregivers: A cross-sectional study in Portugal. Front Med. 2017;4:1-7

25. Lima-Costa MF, Mambrini JVM, Peixoto SV, Malta DC, Macinko J. Socioeconomic inequalities in activities of daily living limitations and in the provision of informal and formal care for noninstitutionalized older Brazilians: National Health Survey, 2013. Int J Equity Health. 2016;15(1):1-8 [acceso el 11 de noviembre del 2020]. Disponible en: http://dx.doi.org/10.1186/ s12939-016-0429-2

26. Lima-Costa MF, Peixoto SV, Malta DC, Szwarcwald CL, Mambrini JVM. Informal and paid care for Brazilian older adults (National Health Survey, 2013). Rev Saude Publica. 2017;51:S1-9.

Manuscrito recibido el 24 de noviembre del 2020. Aceptado para publicación, tras revisión, el 4 de febrero del 2021. 


\section{Functional dependence and support for the older persons in Mexico, 2001-2026}

ABSTRACT Objectives. Identify the factors associated with future functional dependence in the elderly in Mexico and with receiving or not receiving support for basic activities of daily living (ADLs); and project the prevalence of functional dependence in 2026.

Methods. Data from the 2001 National Health and Aging Study (ENASEM) and from the 2012 and 2015 follow-up rounds were used. A multinomial logistic regression model was used to analyze factors associated with future dependence, and a logistic regression model was used for factors associated with receiving or not support. The projected number of older persons with functional dependence in 2026 was based on data from the 2015 ENASEM and on estimated rates from the model of future dependence.

Results. Older people, those with a lower educational level, those with hypertension, arthritis, or diabetes, those who had suffered a stroke or fall, and those with some degree of prior functional dependence had a significantly higher risk of mild or severe dependence and death within 11 years, compared to the reference group. Older people and those with severe dependence had higher odds of receiving support compared to reference groups. By 2026, it is estimated that $18.9 \%$ of older people in Mexico will have mild dependence and $9.3 \%$ will be severely dependent.

Conclusions. Factors associated with future dependence and death were age, educational level, certain chronic diseases, having fallen, and having prior functional dependence; the factors associated with receiving support for basic ADLs were severe dependence and age. It is estimated that the prevalence of dependence will increase 2.1 times over 25 years (2001-2026).

Keywords $\quad$ Activities of daily living; long-term care; aging; Mexico.

\section{Dependência funcional e apoio em idosos no México, 2001-2026}

RESUMO Objetivos. Identificar os fatores associados à dependência funcional futura e a receber apoio para a realização das atividades básicas da vida diária (ABVD) em pessoas idosas e fazer uma projeção da dependência funcional nesta população no México em 2026.

Métodos. Analisaram-se dados obtidos no Estudo Nacional de Saúde e Envelhecimento do México (ENASEM) de 2001 e em rodadas subsequentes da pesquisa de 2012 e 2015. Utilizou-se um modelo de regressão logística multinomial para analisar os fatores associados à dependência funcional futura da pessoa idosa e um outro modelo de regressão logística para analisar os fatores associados a receber apoio. As projeções para 2026 de idosos em situação de dependência foram feitas com base nos dados do ENASEM de 2015 e nas estimativas do modelo de dependência futura.

Resultados. Idade avançada, nível de escolaridade mais baixo, ter hipertensão, artrite, diabetes, história de embolia cerebral ou quedas e possuir algum grau de dependência funcional anterior foram os fatores que se associaram a um risco significativamente maior de dependência funcional (leve ou grave) e morte nos 11 anos seguintes em comparação aos grupos de referência. Idosos com idade avançada ou dependência grave apresentaram uma maior probabilidade de receber apoio para a realização das ABVD em comparação aos grupos de referência. A projeção para 2026 no México é que 18,9\% das pessoas idosas terão dependência leve e 9,3\% dependência grave.

Conclusões. Os fatores associados a dependência funcional futura e morte foram idade, nível de escolaridade, certas doenças crônicas, história de quedas e dependência funcional anterior. Ter idade avançada e dependência grave severa foram os fatores associados a receber apoio para a realização das ABVD. Estima-se que a prevalência de dependência funcional em pessoas idosas aumentará 2,1 vezes em 25 anos (2001-2026).

Palavras-chave Atividades cotidianas; assistência de longa duração; envelhecimento; México. 